\title{
Percepción del Programa Institucional de Tutorías en la Licenciatura en Gestión Tecnológica de la Escuela Superior de Tizayuca
}

\author{
Perception of the Institutional Counseling Program in the Bachelor's Technological \\ Management of the Higher School of Tizayuca
Myriam Coronado-Meneses ${ }^{a}$, Maribel González-Cadena ${ }^{b}$ Elba M. Pedraza-Amador ${ }^{c}$, María F. Montoya-Garrido ${ }^{d}$, Michel Zamorano-Austria ${ }^{e}$, Mayra A. Valerio-Nolasco $^{f}$

\begin{abstract}
:
This work identifies the perception of the students of the Bachelor's Technological Management on the Institutional Counseling Program (ICP), has a non-experimental cross-sectional type with descriptive scope and responds to the need for evaluation of the bachelor's degree with respect to ICP.

The results indicate that the main activities that impact positively are the empathic attitude of the tutors, the cordiality and the tutor's ability to create a climate of trust, the tutor's willingness to serve the students, satisfaction with the tutor assigned to them and the commitment and capacity of the tutor with the tutorial action. However, there are roles should be promoted to improve this function, as the ability to guide students in academic decisions, strengthen the tutor's knowledge of institutional regulation and channel the student to the appropriate instances when they have a problem that go through the tutor's area of action.
\end{abstract}

\section{Keywords:}

Tutor, student, evaluation.

\section{Resumen:}

Este trabajo, que identifica la percepción de los alumnos de la Licenciatura en Gestión Tecnológica sobre el Programa Institucional de Tutorías, posee un diseño no experimental de tipo transversal con alcance descriptivo y responde a la necesidad de evaluación que tiene la licenciatura respecto al PIT.

Los resultados señalan que las principales actividades que impactan de manera positiva son la actitud empática de los tutores, la cordialidad y capacidad del tutor para crear un clima de confianza, la disposición del tutor para atender a los alumnos, la satisfacción con el tutor que le fue asignado y el compromiso y capacidad del tutor con la acción tutorial.; sin embargo, hay funciones que deben fomentarse para mejorar dicha función como la Capacidad para orientar a los alumnos en decisiones académicas, fortalecer el conocimiento del tutor respecto a la normatividad institucional y canalizar al alumno a las instancias adecuadas cuando tiene algún problema que rebasa el área de acción del tutor.

\section{Palabras Clave:}

Tutor, Alumno tutorado, Evaluación.

a Myriam Coronado-Meneses, Universidad Autónoma del Estado de Hidalgo, https://orcid.org/0000-0003-1171-516X, Email:
myriam_coronado3187@uaeh.edu.mx
b Maribel González-Cadena, Universidad Autónoma del Estado de Hidalgo, https://orcid.org/0000-0001-5371-0442, Email:
maribel_gonzalez4257@uaeh.edu.mx
c Elba M. Pedraza -Amador, Universidad Autónoma del Estado de Hidalgo, https://orcid.org/0000-0002-7182-2437, Email:
elbam@uaeh.edu.mx
d María F. Montoya-Garrido, Universidad Autónoma del Estado de Hidalgo, https://orcid.org/0000-0002-7465-108X, Email:
mo356193@uaeh.edu.mx
e Michel Zamorano-Austria, Universidad Autónoma del Estado de Hidalgo, https://orcid.org/0000-0002-4376-0275, Email:
mo356193@uaeh.edu.mx
f Mayra A. Valerio-Nolasco, Universidad Autónoma del Estado de Hidalgo, https://orcid.org/0000-0003-2155-1250, Email: mayra_valerio@uaeh.edu.mx 


\section{Introducción}

Una de las encomiendas que tienen las Instituciones de Educación Superior, a nivel nacional, es establecer acciones que posibiliten la mejora en la calidad de la educación que se brinda a los alumnos durante su formación como profesionistas.

El Plan Sectorial de Educación (PSE) del período actual (2020-2024) indica en la estrategia prioritaria 2.4 que se deben de "Consolidar esquemas de acompañamiento y convivencia escolar orientados a disminuir el abandono escolar y mejorar la eficiencia terminal favoreciendo la transición entre los tipos, niveles y modalidades del Sistema Educativo Nacional" y, específicamente en el apartado 2.4.5, señala que se deben "Implementar programas de tutorías, orientación profesional y nivelación académica, y ampliar la flexibilidad entre las modalidades escolarizada, no escolarizada y mixta, para mejorar la eficiencia terminal, la absorción y el rendimiento escolar en la educación media superior y superior".

En este sentido, el Plan Estatal de Desarrollo del Estado de Hidalgo actual, establece que se debe de "Fortalecer el contenido de programas de tutorías y asesoría académica de todos los niveles educativos para atender con pertinencia la diversidad cultural y lingüística presente en las aulas (PEDH, 2016).

Por su parte la Asociación Nacional de Universidades e Instituciones de Educación Superior (ANUIES) establece en su Programa Institucional de Tutorías (PIT) "Una Propuesta de la ANUIES para su organización y funcionamiento en las instituciones de educación superior" (2001) y recomienda a las universidades agremiadas coordinar programas que ayuden a la formación integral de sus estudiantes para que concluyan sus estudios en tiempo y forma además de evaluar el PIT por medio de metodologías e instrumentos de investigación que coadyuven al conocimiento de la eficacia y la eficiencia del mismo dando principal relevancia al tutor y al estudiante.

La Tutoría, en la Universidad Autónoma del Estado de Hidalgo, (UAEH) inicia en los años ochenta en la Dirección de Orientación Educativa para identificar las particularidades de los alumnos y detectar sus necesidades al ingresar en la institución. En este contexto, en el año 1996, la Dirección de Orientación Educativa, en conjunto con sus docentes tutores, amplían a los estudiantes los beneficios del servicio de tutorías, dirigiendo el servicio también a la retroalimentación del aprendizaje y a la solución de dudas que amplíen el conocimiento del alumnado.
En este sentido y con el objetivo de incrementar la eficiencia terminal de sus programas educativos la Escuela Superior de Tizayuca (ESTi), de la UAEH, instituye, en el año 2005, el Programa de Tutorías en la Ingeniería en Electrónica y Telecomunicaciones y en la Licenciatura en Turismo. En seguimiento a ello y no obstante, que la UAEH contaba con un Programa de Tutorías, en su Modelo Educativo del año 2007, establece el Programa Institucional de Tutorías con el objetivo de identificar y atender las necesidades académicas y personales de los estudiantes para incrementar la eficiencia terminal (UAEH, 2007).

Con el propósito de incrementar su oferta educativa, la ESTi instituye, en el año 2009, la Licenciatura en Gestión Tecnológica y, en atención a los que establece el Modelo Educativo de la UAEH, se brinda a todos sus estudiantes el servicio de tutoría con la finalidad de mejorar indicadores de eficiencia terminal y de disminuir la deserción escolar.

En atención a los lineamientos y recomendaciones que emanan de la Asociación Nacional de Universidades e Instituciones de Educación Superior (ANUIES) respecto al PIT, no se cuenta con una evaluación actual de la percepción de los alumnos respecto a este programa; por lo anterior, se considera prioritario realizar una investigación que brinde seguimiento del impacto de la actividad tutorial durante la formación de alumno de la Licenciatura en Gestión Tecnológica.

\section{Objetivo general}

El presente trabajo tiene como objetivo identificar la percepción de los alumnos de la Licenciatura en Gestión Tecnológica sobre el Programa Institucional de Tutorías que recibe durante su formación.

\section{Materiales y métodos}

Este trabajo de investigación, que ostenta un diseño no experimental de tipo transversal con alcance descriptivo, se aborda a partir de instrumento que propone la ANUIES y consta de dos etapas.

En la primera etapa se realizó investigación documental y en la segunda etapa se aplicó el instrumento de investigación a una muestra de estudiantes adscritos a la Licenciatura en Gestión Tecnológica.

Población y muestra 
Para esta investigación la población se integra por los estudiantes de la Licenciatura en Gestión Tecnológica. Las unidades de análisis se conforman por alumnos que tengan más de un año como estudiantes tutorados. Derivado de los criterios establecidos se determina aplicar muestreo a 47 alumnos del programa educativo.

Para determinar el tamaño de la muestra en poblaciones finitas se adoptaron las recomendaciones de Castañeda, De la Torre, Morán y Lara (2011) y se optó por tolerar un porcentaje de error del $4 \%$ con un nivel de confianza del 95\% para aplicar en la ecuación propuesta por los autores:

$n=\frac{Z^{2} p q N}{N e^{2}+Z^{2} p q}$

En donde:

$\mathrm{n}=$ tamaño de la muestra

$Z^{2}=$ nivel de confianza

$\mathrm{p}=$ variabilidad positiva

$\mathrm{q}=$ variabilidad negativa

$\mathrm{N}=$ tamaño de la población

$e^{2}=$ precisión o error

Al aplicar la ecuación a las unidades de análisis, se determinó el tamaño de la muestra como se aprecia en la Tabla 1.

Tabla 1. Muestra de unidades de análisis para aplicación del instrumento

\begin{tabular}{|l|r|c|}
\hline Unidad de análisis & $\begin{array}{c}\text { Tamaño de } \\
\text { la muestra }\end{array}$ & $\begin{array}{c}\text { Porcentaje } \\
\text { acumulado de la } \\
\text { muestra }\end{array}$ \\
\hline 47 alumnos & 32 & 100.00 \\
\hline
\end{tabular}

Fuente: elaboración propia.

\section{Marco Conceptual}

\subsection{La Tutoría}

Para la ANUIES la tutoría es un proceso de acompañamiento de tipo personal y académico a lo largo del proceso formativo para mejorar el rendimiento académico, solucionar problemas escolares, desarrollar hábitos de estudio, trabajo, reflexión y convivencia social (UNAM 1999).

\section{$1.2 \mathrm{El}$ Tutor}

De acuerdo con el Prontuario de Guía de Trabajo del Tutor de la UAEH (2016) el tutor "es el docente responsable de acompañar, guiar y orientar al estudiante en el proceso académico, personal y laboral para el logro de sus objetivos y el cumplimiento de las demandas profesionales y sociales" (UAEH, 2016).

\subsection{Alumno tutorado}

El alumno tutorado es el estudiante inscrito con base en lo estipulado por la Dirección de Administración Escolar de la $\mathrm{UAEH}$, y por tanto, tiene derecho al beneficio de la tutoría (UAEH, 2016).

\subsection{Programa Institucional de Tutorías}

El Programa Institucional de Tutorías de la Universidad Autónoma del Estado de Hidalgo es una actividad que tiene por objetivo ofrecer diferentes opciones de apoyo a los estudiantes a través de una relación más estrecha entre docentes-alumnos que favorezcan el desarrollo de sus potencialidades y ayudarles a que logren su desarrollo personal, profesional y social. (PIT, 2010).

\subsection{Evaluación}

Un proceso que faculta a las instituciones para mantener sus servicios al día y asegurarse de que se cumplen las necesidades tanto de la organización como de los clientes es la evaluación, la cual, se incorpora en todos los sectores de la actividad humana, entre ellas la educativa, misma que necesariamente para conocer si cumple con su encomienda se debe someter a un tipo de valoración a fin de conocer los resultados que tiene y en su caso tomar decisiones que le permitan reorientar su actividad. La evaluación pretende en general, conocer y valorar no sólo los resultados que obtiene, sino también la relación entre éstos y los medios utilizados.

Stufflebeam \& Shinkfield (1987) citan la definición del Joint Committee on Standards for Educational Evaluation que refiere a la evaluación como "el enjuiciamiento sistemático de la valía o el mérito de un objeto". Esta enunciación supone un juicio que emplea procedimientos objetivos que deriven en información imparcial para determinar qué tan bueno o malo es el objeto que se enjuicia.

Para Stufflebeam \& Shinkfield (1987) la valoración desempeña un papel fundamental que sirve para identificar puntos débiles y fuertes con el objetivo de tender hacia una mejora, los autores proponen que es 
importante considerar los siguientes criterios al momento de realizar una evaluación:

Las expectativas de los clientes.

Que haga bien lo que se supone que debe hacer.

Determinar hasta qué punto el servicio es necesario.

\section{Resultados}

Para responder al objetivo de este estudio, en este apartado, se presentan los resultados que, de acuerdo con la percepción estudiantes tutorados, coadyuvan al impacto positivo de la actividad tutorial, así como aquellas funciones que requieren fortalecerse para contribuir a los lineamientos que establece el Programa Institucional de Tutorías.

Cabe señalar que se estableció como criterio aceptable, para la evaluación de las actividades del PIT, la valoración de competencias a partir del $70 \%$ en base a que su equivalente en calificación numérica (siete) se establece en el Modelo Curricular Integral 2007 como suficiente en la adquisición de un contenido, competencia u otra conducta susceptible de ser medida y calculada (UAEH, 2007).

\section{A. Actitud empática}

El $93.75 \%$ de los alumnos manifiesta estar totalmente de acuerdo en que el tutor los Trata con respeto y atención; al mencionarles si La cordialidad y capacidad del tutor logra crear un clima de confianza para que pueda exponer sus problemas, un $81.25 \%$ de ellos está totalmente de acuerdo, no obstante, el $6.25 \%$ indicó estar más o menos de acuerdo.

B. Compromiso con la Actividad tutorial

Con referencia a si El tutor muestra capacidad para escuchar los problemas de los alumnos, el $87.5 \%$ señala estar totalmente de acuerdo, sin embargo, el $12.5 \%$ indicó estar más o menos de acuerdo.

Al preguntar a los estudiantes si Muestra el tutor interés en los problemas académicos y personales que afectan el rendimiento de los alumnos, el $87.5 \%$ señaló estar totalmente de acuerdo, el $6.25 \%$ manifestó estar de acuerdo y el $6.25 \%$ señaló estar más o menos de acuerdo.

C. Capacidad para la Acción tutorial

En conjunto, el $81.25 \%$ de los estudiantes señalan estar totalmente de acuerdo en que el tutor Tiene capacidad para resolver sus dudas académicas, en que Tiene capacidad para diagnosticar las dificultades y que realiza las acciones pertinentes para resolverlas; el $87.5 \%$ está Totalmente de acuerdo respecto a si el tutor Tiene capacidad para orientarlos en metodología y técnicas de estudio, Tiene capacidad para estimular el estudio independiente, que Posee formación profesional en su especialidad y que
Posee métodos pedagógicos para la atención individualizada o grupal.

D. Disposición para atender a los alumnos

Los resultados de investigación exhiben que, en conjunto, el $93.75 \%$ de los alumnos están totalmente de acuerdo en que su tutor Muestra buena disposición para atenderlos; en que Es fácil localizar al tutor que tiene asignado por las sesiones agendadas y el $81.25 \%$ exhibe estar totalmente de acuerdo en que su tutor Muestra disposición a mantener una comunicación permanente, mientras que el $18.75 \%$ comenta estar más o menos de acuerdo.

E. Capacidad para orientar a los alumnos en decisiones académicas.

La percepción, en conjunto, del $75 \%$ de los alumnos indica que están totalmente de acuerdo y $25 \%$ de acuerdo en que su tutor Conoce suficientemente la normatividad institucional para aconsejarle las opciones adecuadas a sus problemas escolares; al solicitar su percepción sobre si El tutor lo canaliza a las instancias adecuadas cuando tiene algún problema que rebasa su área de acción, el $75 \%$ de los estudiantes está totalmente de acuerdo y el $25 \%$ señaló estar más o menos de acuerdo; y por último, el $87.5 \%$ de los alumnos está de acuerdo en que La orientación recibida de parte del tutor le ha permitido realizar una selección adecuada de cursos y créditos.

\section{F. Satisfacción}

El $87.5 \%$ de los estudiantes está totalmente de acuerdo en que El tutor que le fue asignado es adecuado y que Es satisfactorio el programa de tutoría.

\section{Conclusiones}

En relación a la evaluación de la función tutorial, por parte de los estudiantes tutorados de la Licenciatura en Gestión Tecnológica, los principales resultados del instrumento de investigación aplicado señalan que existen funciones que impactan de manera positiva la actividad tutorial y funciones que deben fortalecerse para mejorar dicha actividad.

Funciones impactan de manera positiva de mayor a menor:

- La Actitud empática de los tutores en el trato con respeto y atención y La cordialidad y capacidad del tutor para crear un clima de confianza.

- La Disposición del tutor para atender a los alumnos.

- La satisfacción con el tutor que le fue asignado y con el PIT.

- Compromiso con la Actividad tutorial.

- La Capacidad para la acción tutorial. 


\section{Funciones deben fomentarse para mejorar dicha actividad}

La Capacidad para orientar a los alumnos en decisiones académicas, principalmente en:

- Fortalecer el conocimiento del tutor respecto a la normatividad institucional para aconsejarle las opciones adecuadas a sus problemas escolares

- Canalizar al alumno a las instancias adecuadas cuando tiene algún problema que rebasa su área de acción

\section{Referencias}

[1] Asociación Nacional de Universidades e Institutos de Educación Superior. [ANUIES]. (1999).

[2] Castañeda, J., de la Torre, M., Morán, J., \& Lara, L. (2011) Metodología de la investigación. México: McGraw Hill.

[3] Gobierno del Estado de Hidalgo. (2016). Plan Estatal de Desarrollo del Estado de Hidalgo Recuperado de http://planestataldedesarrollo.hidalgo.gob.mx/docs/PlanEstatal.pdf

[4] Programa Institucional de Tutorías, Una propuesta de la ANUIES para su organización y funcionamiento en las instituciones de educación superior, ANUIES. (2001). Recuperado de: http://www.anuies.mx/servicios/d_estrategicos/libros/lib42/000.htm

[5] Dirección General de Servicios Estudiantiles, Programa Institucional de Tutorías (2010). Universidad Autónoma del Estado de Hidalgo.

[6] Secretaria de Educación Pública [SEP]. (2020). Plan Sectorial de Educación. Recuperado de https://www.gob.mx/cms/uploads/attachment/file/562380/Programa S ectorial_de_Educaci_n_2020-2024.pdf

[7] Stufflebeam, D., \& Shinkfield, A. (1987). Evaluación sistemática. Guía teórica y práctica. Barcelona: Paidos.

[8] Universidad Autónoma del Estado de Hidalgo [UAEH]. (2007). Modelo Curricular Integral UAEH. Reforma del Nivel Licenciatura. Pachuca, Hidalgo. 